\title{
Multicentric and multifocal versus unifocal breast cancer: differences in the expression of E-cadherin suggest differences in tumor biology
}

Tobias Weissenbacher ${ }^{1,6^{*}}$, Eva Hirte ${ }^{1}$, Christina Kuhn ${ }^{1}$, Wolfgang Janni ${ }^{2}$, Doris Mayr ${ }^{3}$, Uwe Karsten ${ }^{5}$, Brigitte Rack , Klaus Friese ${ }^{1}$, Udo Jeschke ${ }^{1}$, Sabine Heublein ${ }^{1}$, Darius Dian ${ }^{1}$ and Nina Ditsch ${ }^{4}$

\begin{abstract}
Background: The aim of this study was to evaluate the expression of the cell adhesion-related glycoproteins MUC-1, $\beta$-catenin and E-cadherin in multicentric/multifocal breast cancer in comparison to unifocal disease in order to identify potential differences in the biology of these tumor types.

Methods: A retrospective analysis was performed on the expression of $\mathrm{MUC1}$, $\beta$-catenin and E-cadherin by immunohistochemistry on tumor tissues of a series of 112 breast cancer patients (total collective) treated in Munich between 2000 and 2002. By matched-pair analysis, 46 patients were entered into two comparable groups of 23 patients after categorizing them as having multicentric/multifocal or unifocal breast cancer. Matching criteria were tumor size, histology grade and lymph node status; based on these criteria, patients were distributed equally between the two groups ( $p=1.000$ each). Data were analyzed with the Kruskal-Wallis and the Mann-Whitney tests.

Results: In the matched groups, we found a significantly down-regulated expression of E-cadherin in multicentric/multifocal breast cancer compared to unifocal disease $(p=0.024)$. The total collective showed even higher significance with a value of $p<0.0001$. In contrast, no significant differences were observed in the expression of $\beta$-catenin between multicentric/multifocal and unifocal tumors ( $p=0.636$ and $p=0.914$, respectively). When comparing the expression of MUC1, E-cadherin and $\beta$-catenin within the unifocal group, we found a significant positive correlation between E-cadherin and $\beta$-catenin $(p=0.003)$. In the multicentric/multifocal group we observed, in contrast to the unifocal group, a significant decrease of MUC1 expression with increased grading $(p=0.027)$.

Conclusion: This study demonstrates that multicentric/multifocal and unifocal breast cancers with identical TNM-staging clearly differ in the expression level of E-cadherin. We suggest that the down-regulation of E-cadherin in multicentric/multifocal breast cancer is causally connected with the worse prognosis of this tumor type.
\end{abstract}

Keywords: Breast cancer, MUC-1, Multicentric, Multifocal, Tumor biology, E-cadherin, $\beta$-catenin

\section{Background}

Tumor-node-metastasis (TNM) staging has been the standard method for breast cancer classification for more than fifty years. During this time, however, the classification procedure has changed substantially. In 2003, the 6th edition of the TNM classification was established [1-3]. The T category has maintained its prognostic relevance throughout

\footnotetext{
* Correspondence: tobias.weissenbacher@med.uni-muenchen.de ${ }^{1}$ Frauenklinik, Klinikum der Ludwig-Maximilians-Universität, Innenstadt, München, Germany

${ }^{6}$ Department of Gynecology and Obstetrics, Campus Innenstadt LudwigMaximilian-University Munich, Maistr. 11, Munich D-80337, Germany Full list of author information is available at the end of the article
}

these changes [3]. The prognosis of breast cancer patients depends on two different types of factors: tumor size as a time-dependent marker of tumor biology, and biological factors (i.e., histological grade) which represent tumor aggressiveness [4]. Other prognostic factors include the estrogen and progesterone receptor status as well as the relative number of mitotic figures (MF/10HPF) [5,6]. Treatment plans are following worldwide prevailing suggestions, including those of the TNM system. However, the TNM classification has changed, and treatment recommendations and the treatments themselves have been modified. Breast-conserving treatment, once a controversial issue, is

\section{Biomed Central}

(c) 2013 Weissenbacher et al.; licensee BioMed Central Ltd. This is an Open Access article distributed under the terms of the Creative Commons Attribution License (http://creativecommons.org/licenses/by/2.0), which permits unrestricted use, distribution, and reproduction in any medium, provided the original work is properly cited. 
now an established alternative to modified radical mastectomy for surgically manageable breast cancer.

In a recent study we have demonstrated that focality is an independent prognostic factor by comparing multicentric/multifocal and unifocal breast cancer [7]. Therefore, additional biological factors seem to play an important but not well understood role in multicentric/ multifocal breast cancers.

The above-mentioned established prognostic factors $[4,8,9]$ as well as potential new factors, such as the E-cadherin-related transcriptional repressor Snail or the c-Jun activation domain-binding protein-1 (Jab1), are multifunctional signaling proteins. The E-cadherin/ catenin complex is known to be a potent inhibitor of cancer progression [10-13].

The disconnection of cell-cell adhesions is a fundamental step in the progression of cancer and metastasis that is mediated by a variety of membrane proteins. The transmembrane protein E-cadherin, which is responsible for calcium-dependent cell adhesions, is a widely studied tumor suppressor. It is expressed predominantly in epithelial cells, and its extracellular region has a $\mathrm{Ca}^{2+}$-dependent homophilic adhesion function. Loss of E-cadherin has been reported to induce epithelial-mesenchymal transition in several cancers [14-16].

Epithelial mucin-1 (MUC1) is a complex transmembrane glycoprotein. The larger, heavily glycosylated domain of the MUC molecule is extracellularly expressed [17]. MUC1 exerts a number of different functions [18-23]. MUC1 undergoes characteristic modifications of its glycosylation and cellular localization during malignant transformation [24]. Many monoclonal antibodies have been developed to MUC1 [17]. A novel antibody, PankoMab, was developed against a tumor-associated epitope of MUC1 [19]. In a previous paper, PankoMab was examined in patients with breast cancer in comparison with two other known antibodies. PankoMab was unique to the effect that its staining was correlated with the estrogen receptor expression [20].

The glycoprotein $\beta$-catenin interacts with both Ecadherin and MUC1. The interaction between MUC1 and E-cadherin is mediated by $\beta$-catenin-binding and interrupts E-cadherin-mediated cell-cell adhesions. Signal transduction through $\beta$-catenin (the so-called Wnt/ $\beta$-catenin signaling pathway) has already been thoroughly investigated [21]. This signal transduction regulates the expression of a number of genes essential for cell differentiation and proliferation. Alterations in this pathway are implicated in diseases such as cancer [22].

The aim of this study was to compare the expression of MUC1, E-cadherin and $\beta$-catenin in multicentric/ multifocal tumors with their expression in unifocal tumors of identical tumor size according to TNM staging in order to detect potential differences.

\section{Methods}

\section{Patients}

Two groups were framed and investigated. Based on a consecutive patient cohort consisting of 112 patients documented and surgically treated for primary breast cancer between 2000 and 2002 at the Department of Gynecology of the University Hospital in MunichInnenstadt, 57 unifocal breast cancer patients and 55 patients with multicentric/multifocal disease formed our total collective (TC). From the same patient cohort, two equivalent groups of 23 breast cancer patients with multicentric/multifocal vs. unifocal tumors were selected using a matched paired analysis (MG) (see Statistical Analysis section below). The Institutional Review Board of the Ludwig Maximilians University Munich, Germany, approved the study and all the patients gave informed consent.

Unifocality versus multicentricity/multifocality were determined by clinical examination, ultrasound and X-ray. In addition, in a few cases nuclear magnetic resonance imaging (NMRI), galactography or pneumocystography was performed if necessary. These techniques were used in a few cases, in which additional information regarding focality was necessary. Moreover, those cases which failed to confirm multicentricity/multifocality with respect to the final histological examination were excluded.

Data were contemporaneously gathered for the unifocal and multicentric/multifocal tumors. To be eligible, patients were required to be free of disease, and they must have been treated at the study site at the time of primary diagnosis of resectable breast cancer. The tumor stage at primary diagnosis was classified according to the UICC TNM classification [23]. Tumor grading by WHO (Nottingham grading respectively to Elston \& Ellis modification of Bloom-Richardson grading [25] was used, and match criteria were tumor size, histology grade and lymph node status, all of which were equally distributed between the two groups $(\mathrm{p}=1.0)$. The total collective was not matched. We used this group to validate the results of the matched group.

\section{Surgical treatment}

The primary surgical treatment consisted of either breast conservation or modified radical mastectomy. Routine axillary dissections were performed on levels I and II lymph nodes, while level III lymph nodes were only excised in cases expressing macroscopic metastatic lesions of the lower levels. For the diagnosis of lymph node metastasis, single embedded lymph nodes were screened at up to three levels.

The guidelines for chemotherapy and cytostatic regimes changed substantially also within the observation time of the study. Therefore the authors did not include oncological treatment details. 


\section{Immunohistochemistry}

Immunohistochemistry was performed using a combination of pressure cooker heating for antigen retrieval and the standard streptavidin-biotin-peroxidase complex with the use of the mouse IgG-Vectastain Elite ABC kit (Vector Laboratories, Burlingame, CA, USA). Table 1 lists the mouse monoclonal antibodies used for these experiments.

Formalin-fixed paraffin embedded tissue sections were dewaxed using xylol for $15 \mathrm{~min}$, rehydrated in an descending series of alcohols (100\%, 96\%, and 70\%), and subjected to epitope retrieval for $5 \mathrm{~min}$ in a pressure cooker using sodium citrate buffer ( $\mathrm{pH}$ 6.0). After cooling, sections were washed twice in PBS. Endogenous peroxidase activity was quenched by immersion in $3 \%$ hydrogen peroxide in methanol for $20 \mathrm{~min}$. Non-specific binding of the primary antibodies was blocked by pretreatment of the sections with diluted normal serum (10 ml PBS containing $150 \mu \mathrm{l}$ horse serum; Vector Laboratories, Servion, Switzerland) for $20 \mathrm{~min}$. Sections were then incubated with the primary antibodies at room temperature for $60 \mathrm{~min}$. After washing with PBS, sections were incubated in diluted biotinylated secondary antiserum (10 ml PBS containing $50 \mu \mathrm{l}$ horse serum; Vector Laboratories) for $30 \mathrm{~min}$ at room temperature. After incubation with the avidin-biotin peroxidase complex (diluted in $10 \mathrm{ml}$ PBS, Vector Laboratories) for $30 \mathrm{~min}$ and repeated washing steps with PBS, visualization was performed with DAB substrate (Dako, Glostrup, Denmark) for $2 \mathrm{~min}$. Sections were counterstained with Mayer's hematoxylin and dehydrated in an ascending series of alcohols (50-98\%), followed by xylol. Finally, sections were embedded, but mounted and covered. Negative controls were performed by replacing the primary antibody with normal horse serum. Immunohistochemical staining was performed using an appropriate positive control.

The intensity and distribution patterns of specific immunohistochemical staining were evaluated using the semi-quantitative immuno-reactive score (IRS). This score was calculated by multiplying the staining intensity (graded as 0 = no, 1 = weak, $2=$ moderate and $3=$ strong staining) with the percentage of positively stained cells $(0=$ no staining, $1=<10 \%$ of cells, $2=11-50 \%$ of cells,

Table 1 Antibodies employed

\begin{tabular}{|c|c|c|c|c|}
\hline Antigen & Antibody/clone & Isotype & Dilution & Source \\
\hline E-cadherin & HECD-1 & $\begin{array}{l}\text { Mouse } \\
\operatorname{lgG} 1\end{array}$ & $1: 80$ & $\begin{array}{l}\text { Merck, Darmstadt, } \\
\text { Germany }\end{array}$ \\
\hline$\beta$-catenin & polyclonal & $\begin{array}{l}\text { Rabbit } \\
\lg G\end{array}$ & $1: 100$ & $\begin{array}{l}\text { Diagnostic BioSystems, } \\
\text { Pleasanton, CA, USA }\end{array}$ \\
\hline MUC1 & mPankoMab & $\begin{array}{l}\text { Mouse } \\
\operatorname{lgG} 1\end{array}$ & $1: 550$ & $\begin{array}{l}\text { Glycotope, } \\
\text { Berlin, Germany }\end{array}$ \\
\hline
\end{tabular}

$3=51-80 \%$ of cells and $4=>81 \%$ of cells stained). The slides were examined by two independent observers. Sections were examined using a Leitz microscope (Wetzlar, Germany) with a 3CCD color camera (JVC, Victor Company of Japan, Japan).

\section{Statistical analysis}

Data were entered into the database in a coded fashion. Our total collective of 112 patients included 57 unifocal breast cancer patients and 55 cases of multicentric/ multifocal tumors. Because of the uneven distribution of prognostic factors in our original patient group of 46 cases that met the match criteria, a matched pair analysis was performed. A total of 23 pairs of patients, each consisting of one patient with unifocal and one with multicentric/multifocal tumor lesions, were selected according to the highest degree of equivalence in the following hierarchical and sequential order: tumor size at the time of primary diagnosis, histology grading, and lymph node status. Each parameter was required to have a $\mathrm{p}$ value $>0.50$ to achieve intergroup homogeneity. We deliberately matched patients based on the criteria at the time of primary diagnosis. The computer software 'Statistical Package for the Social Sciences 15.0' (SPSS Inc., Chicago, IL, USA) was used to perform statistical analyses. We used Kruskal-Wallis one-way analysis of variance to analyze our data, which is a non-parametric method for testing equality of population medians among groups. It is an extension of the Mann-Whitney U test to 3 or more groups.

For survival analysis median immunoreactivity levels, as determined by the IR-score, of each marker were employed to split the collective into low vs. high expressing cases. The following thresholds were used: E-Cadherin $\geq \operatorname{IRS} 8$, beta-Catenin (membrane staining) $\geq$ IRS 8 , beta-Catenin (cytoplasma staining) $\geq$ IRS 4, MUC1 (membrane staining) $\geq$ IRS 8, MUC1 (cytoplasma staining) $\geq$ IRS 1. Kaplan-Meier survival curves were drawn to compare survival times of uni- vs. multifocal/-centric tumors and of high vs low expressing cases, respectively. Differences in overall and relapse-free survival were tested for significance by applying the chi-square statistic of the log rank test.

$P$ values below 0.05 were considered significant.

\section{Results}

All matching criteria (tumor size, histology grade and lymph node status) were equally distributed between the two groups $(\mathrm{p}=1.0)$.

No significant difference was observed between the two groups in terms of age ( $p=0.104$ in the matched group and $\mathrm{p}=0.533$ in the total collective) or menopausal status (MG: $\mathrm{p}=0.291$ and TC: $\mathrm{p}=0.503$ ). Regarding histological types of tumors, the total collective (TC) demonstrated a statistically significant difference with $\mathrm{p}=0.003$ (see below), whereas no significant difference was found in 
the matched group $(\mathrm{p}=0.120)$. Table 2 shows the primary patient characteristics of both groups.

Looking at the total collective, 55 patients were included in the multicentric/multifocal group and 57 in the unifocal

Table 2 Patient characteristics

\begin{tabular}{|c|c|c|c|}
\hline \multicolumn{4}{|c|}{ Total collective } \\
\hline & $\begin{array}{c}\text { Multicentric/multifocal } \\
(\%)\end{array}$ & $\begin{array}{l}\text { Unifocal } \\
(\%)\end{array}$ & P-value \\
\hline Number of patients & 55 & 57 & \\
\hline Age & 60.6 & 58.9 & .533 \\
\hline $\begin{array}{l}\text { Lymph node } \\
\text { Metastases }\end{array}$ & & & .150 \\
\hline Absent (N0) & $27(50.0)$ & $35(62.5)$ & \\
\hline 1-3 axillary LNM (pN1bi) & $4(7.4)$ & $7(12.5)$ & \\
\hline 1-3 axillary LNM (pN1biii) & $18(33.3)$ & $8(14.3)$ & \\
\hline 1-3 axillary LNM (pN1biv) & 0 & $3(5.4)$ & \\
\hline 4-9 axillary LNM (pN2) & $1(1.9)$ & $1(1.8)$ & \\
\hline Unknown (pNx) & $5(9.1)$ & $3(5.3)$ & \\
\hline Histological Type & & & .003 \\
\hline Ductal & $35(66)$ & 39 (69.6) & \\
\hline Lobular & $11(20.8)$ & $3(5.4)$ & \\
\hline Ductal-lobular & $4(7.5)$ & $3(5.4)$ & \\
\hline Mucinous & $1(1.9)$ & $2(3.6)$ & \\
\hline Medullary & $1(1.9)$ & $4(7.1)$ & \\
\hline Micropapillary & $1(1.9)$ & $2(3.6)$ & \\
\hline Tubulary & 0 & $3(5.4)$ & \\
\hline Not specified & $2(3.6)$ & $1(1.8)$ & \\
\hline Menopausal Status & & & .503 \\
\hline Premenopausal & $13(47.9)$ & $16(37.5)$ & \\
\hline Postmenopausal & $37(52.1)$ & $36(62.5)$ & \\
\hline \multicolumn{4}{|c|}{ Matched Group } \\
\hline & $\begin{array}{c}\text { Multicentric/multifocal } \\
(\%)\end{array}$ & $\begin{array}{c}\text { Unifocal } \\
(\%)\end{array}$ & P-value \\
\hline Number of patients & 23 & 23 & \\
\hline Age & 57 & 68 & .104 \\
\hline Histological Type & & & .120 \\
\hline Ductal & $16(69.6)$ & $15(65.2)$ & \\
\hline Lobular & $5(21.7)$ & $3(13.0)$ & \\
\hline Ductal-lobular & $2(8.7)$ & $1(4.3)$ & \\
\hline Medullary & 0 & $1(4.3)$ & \\
\hline Micropapillary & 0 & $2(8.7)$ & \\
\hline Not specified & 0 & $1(4.3)$ & \\
\hline Menopausal Status & & & .291 \\
\hline Premenopausal & $4(18.8)$ & $6(26.1)$ & \\
\hline Perimenopausal & 0 & $1(4.3)$ & \\
\hline Postmenopausal & $18(81.8)$ & $14(60.9)$ & \\
\hline Unknown & $1(4.3)$ & $2(8.7)$ & \\
\hline
\end{tabular}

group. This group was not matched, so statistical analysis was performed according the matching criteria of tumor size, lymph node status and histopathological grading. Tumor size $(\mathrm{p}=0.113)$, lymph node involvement $(\mathrm{p}=0.150)$, and histopathological grading $(\mathrm{p}=0.068)$ did not show any significant correlation with multicentric/ multifocal tumors versus unifocal tumors.

According to the histological tumor type, a significant difference was observed in the incidence of invasive lobular cancer in the multicentric/multifocal group in comparison to the unifocal group. Of 14 patients suffering from invasive lobular cancer, 11 had multicentric/multifocal disease, whereas only 3 had unifocal breast cancer. The results were different for invasive ductal tumors; out of 74 patients with invasive ductal cancer, 35 had multicentric/multifocal disease, and 39 had unifocal breast cancer. Looking at the matched group, five patients had lobular multicentric/ multifocal breast cancer (21.7\%), and three patients (13.6\%) had a lobular unifocal disease. Also, ductal carcinomas did not differ significantly. Sixteen patients (69.6\%) in the multicentric/multifocal matched group had ductal breast cancer, compared with 15 patients (68.2\%) in the unifocal group.

Regarding the expression of E-cadherin, lobular cancers were not included in the statistical analysis of the two groups. The total collective examined therefore included 54 unifocal and 44 multicentric/multifocal cancer tissues. Compared to the multicentric/multifocal group, E-cadherin expression was significantly higher in the unifocal group, with a p-value of $<0.0001$. MG in this case included 32 patients (16 pairs). E-cadherin expression was also significantly higher in the unifocal matched group with $\mathrm{p}=0.024$ (Figure 1).

Looking at the grading within the total collectivegroup as well as unifocal tumors, G2 (moderately differentiated) tumors exhibited higher E-cadherin expression compared to multifocal tumors $(\mathrm{p}=0.001)$, as did G3 (poorly differentiated) tumors $(\mathrm{p}=0.037)$. The matched pair group underlined these results for G2 tumors and revealed higher E-cadherin expression in unifocal tumors compared with multicentric/multifocal tumors. The p-value was 0.055 for G2 tumors, whereas G3 tumors failed to demonstrate significance with $\mathrm{p}=0.261$ (Figure 1).

No significant differences in $\beta$-catenin expression patterns were observed between multicentric/multifocal and unifocal tumors $(\mathrm{p}=0.914)$ when comparing the total collective, and the difference was also not significant for the matched pairs $(p=0.636)$. Furthermore, $\beta$-catenin expression showed no significant correlation with histology grade within the total collective either for multicentric/ multifocal breast cancers $(\mathrm{p}=0.564)$ or for unifocal disease (data not shown, $\mathrm{p}=0.635$ ).

However, the cytoplasm $\beta$-catenin was associated significantly with a reduced overall survival (OS) in unifocal 


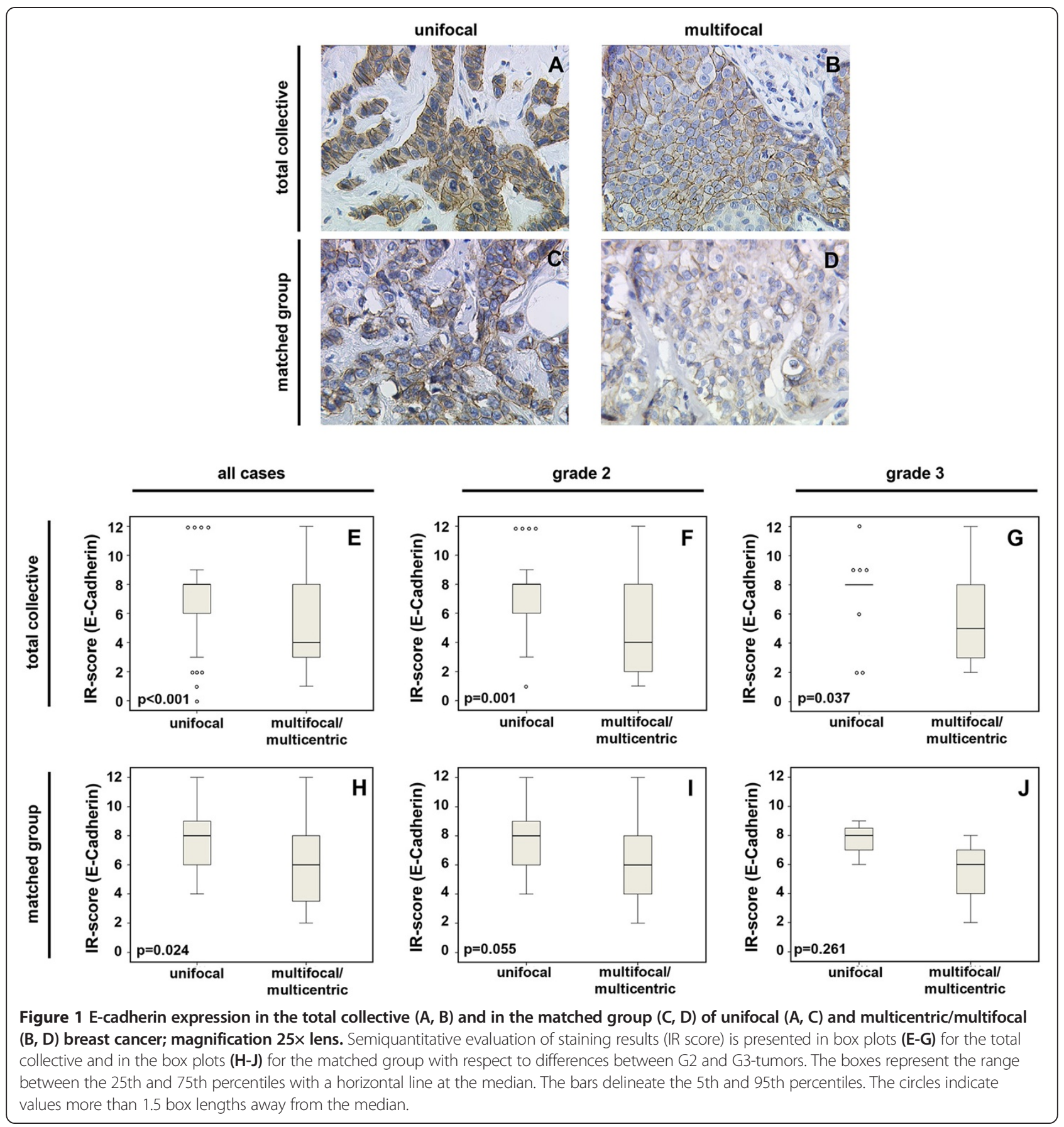

tumors $(p=0.032)$. Interestingly, no differences were found concerning survival in mulicentric/multifocal tumors (Figure 2A).

The MUC1 expression also failed to demonstrate a significant difference between unifocal and multicentric/ multifocal disease in both the MG $(\mathrm{p}=0.840)$ and the TC group $(\mathrm{p}=0.183)$.

Analyzing differences with respect to histology grade, no differences in MUC1 expression were observed in the total collective among G1, G2 and G3 unifocal tumors $(\mathrm{p}=0.840)$. In contrast, MUC1 expression in multicentric/multifocal tumors was significantly dependent on histology grade (decreasing from G1 to G3 at $\mathrm{p}=0.027$ ) (Figure 3).

The PankoMab epitope demonstrated no difference according to the histology grade when looking at the cytoplasm staining. When looking at the overall survival (OS), the PankoMab epitope on the membrane was associated with a better outcome, however only significant in G2 and G3-unifocal tumors (Figure 2B). 

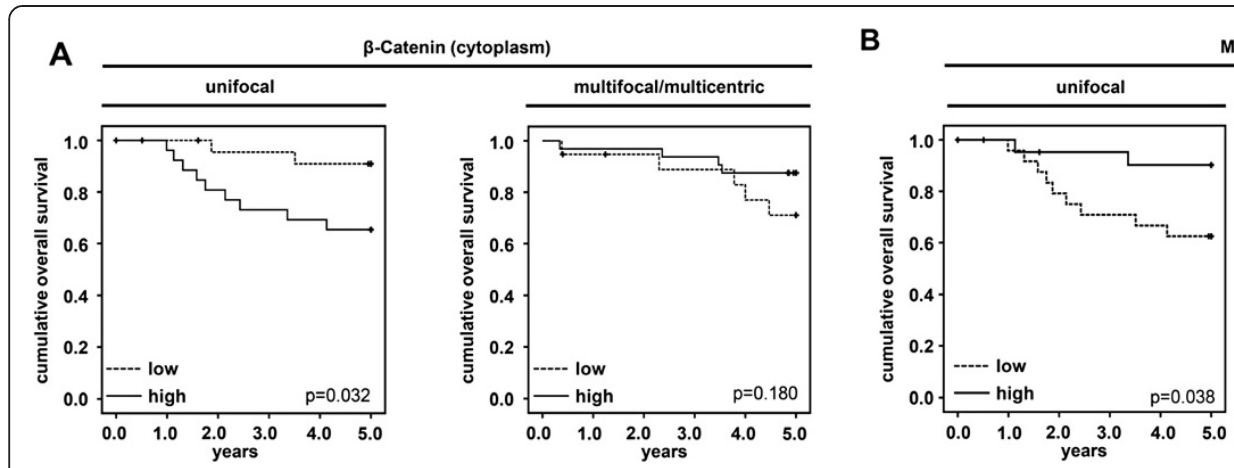

MUC1 (membrane)

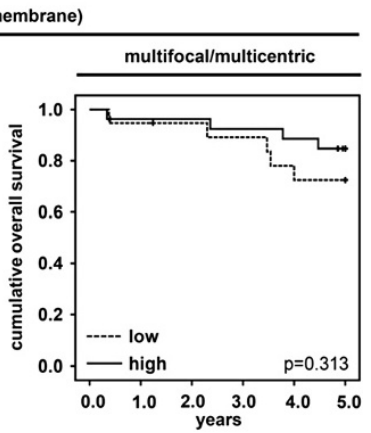

Figure 2 B-Catenin and MUC1 expression related to overall survival and focality. A: Cytoplasmic B-catenin expression related to the overall survival (OS) in unifocal and multicentric/multifocal tumors. B: PankoMab epitope on the membrane related to the overall survival (OS) in unifocal and multicentric/multifocal tumors.

In other words, less differentiated multicentric/multifocal tumors exhibited partial loss of MUC1 expression.

\section{Discussion}

We investigated in a previous study the prognostic differences between multicentric/multifocal and unifocal breast cancer [7]. In that study, patients were entered by matched-pair analysis into two comparable groups of 288 patients after categorizing them as having multicentrical/ multifocal or unifocal breast cancers. Matching criteria were tumor size, histology grade and hormone receptor status, which were equally distributed between both groups ( $p=1.000$ each). We demonstrated that multicentric/multifocal breast cancer is associated with a worse prognosis compared to unifocal disease with an identical tumor size
[7]. However, Vlastots et al. investigated breast cancer patients with early-stage disease and did not find an increased risk of poor outcome with respect to multicentricity. According to the authors, this study supports the current tumor, node, metastasis staging system [26].

On the contrary, Tot et al. also demonstrated recently, that multifocality represents a negative prognostic parameter associated in this study with significantly increased lymphnode metastasis (LNM) [27]. These findings were confirmed by Tot et al. in further studies, that demonstrated multifocality being associated with an increased risk of LNM [28,29].

According to our study-collective of 112 patients, 55 patients were included in the multicentric/multifocal
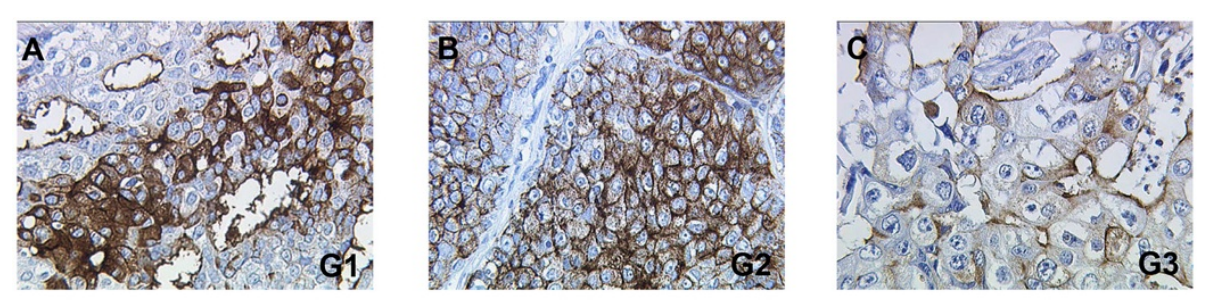

D

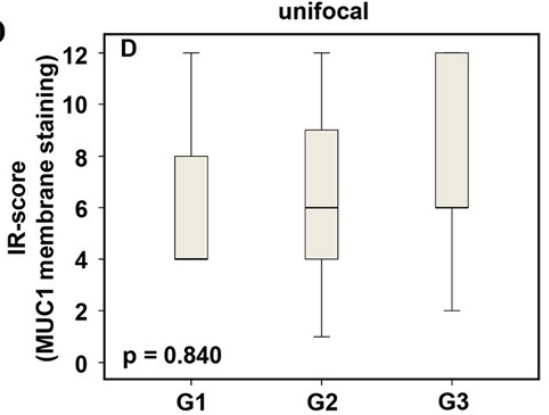

E

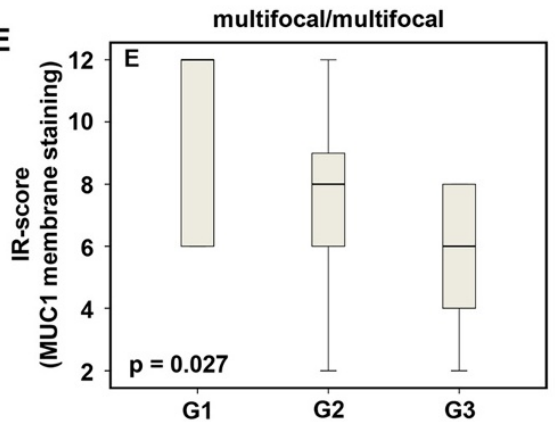

Figure 3 MUC1 (mPankoMab) membrane expression in the total collective of multicentric/multifocal breast cancer in a G1 tumor (A), a G2 tumor (B), and a G3 tumor (C); magnification $\mathbf{2 5} \times$ lens. The box plots (D, E) present a semiquantitative evaluation of staining results (IR score). The boxes represent the range between the 25 th and 75 th percentiles with a horizontal line at the median. The bars delineate the 5th and 95 th percentiles. 
group and 57 in the unifocal group. This total collective was not matched, and statistical analysis was performed according the matching criteria of tumor size, lymph node status and histopathological grading. Our results did however not demonstrate any significant correlation of lymph node metastasis when comparing multicentric/ multifocal and unifocal tumors. This result however has to be interpreted in a critical manner to the effect that the total collective however includes patients who were matched according to the lymph nodes status.

However, it remained unclear whether the tendency of breast cancer tumors to metastasize was a reflection of the total tumor load or whether biological differences play a decisive role. The 10 -year survival rate was investigated by Boyages et al. who found - especially in tumors $>2 \mathrm{~cm}$ - that the aggregate size of every focus should be considered along with other prognostic factors when comparing multifocal and unifocal breast cancer [30].

Aim of this manuscript was, to evaluate differences in tumor biology, that might help explaining the above mentioned differences. Tot et al. investigated multifocal and unifocal breast cancer according to the immunophenotype (estrogen and progesterone receptor expression, HER2 overexpression and expression of basal-like markers, CK5/6, CK14, and epidermal growth factor receptor). The auhors found higher rates of LNM in the multifocal group, interestingly no differences with respect to molecular phenotype [29]. These findings were underlined by Pekar et al. who also demonstrated that diffuse or multifocal distribution of the invasive component is associated with cancer-related death independent of the molecular phenotype [31].

Bassarova et al. [32] investigated the cadherin/catenin immunophenotype of multicentric tumor foci and bilateral breast cancer. They found a greater similarity of the primary tumor to its corresponding metastatic tumor than to the contralateral primary tumor regarding the cadherin/ catenin immunophenotype [32]. Although different histological subtypes were examined (pleomorphic lobular, invasive ductal of usual type, atypical medullary carcinomas, mucinous and invasive micro papillary carcinomas), differences in the tumor biology were obvious and could be anticipated. The present study was intended to analyze some of the potential factors involved.

$\beta$-catenin is involved in cell-cell adhesions and is a transcriptional regulator in the Wnt signaling pathway [33], furthermore it is consequently involved in the development of human malignancies. Lopez-Knowles et al. [34] investigated immunohistochemically the expression of $\beta$-catenin in 292 patients with invasive ductal breast cancers. The authors demonstrated an association between a high cytoplasmic expression of $\beta$-catenin and a high tumor grade $(\mathrm{p}=0.004)$ and negative estrogen receptor values $(p=0.005)$, and the high expression of $\beta$-catenin was thus associated with an adverse disease outcome.

We found no differences for the cytoplasmic $\beta$-catenin as well as for the membrane $B$-catenin with respect to the grading. Moreover, the cytoplasmic $\beta$-catenin was associated significantly with a reduced OS in unifocal tumors $(\mathrm{p}=0.032)$. Our data suggest a wnt signaling pathway in unifocal tumors. However, this pathway might not play an important role in multicentric/multifocal tumors. Therefore we assume differences in tumor biology between uni- and multifocal tumors according to our results.

Niu et al. described an association between abnormal $\beta$-catenin expression, positive lymph node status and high histological grade $(\mathrm{p}<0.01)$ as well as a significant correlation between positive Her2 expression and abnormal $\beta$-catenin expression [13]. Therefore, elevated $\beta$-catenin expression appears to be linked with worse outcome for the patients. However, differences concerning focality have not been investigated.

Recent research has underlined the importance of E-cadherin with respect to cell adhesion mechanisms. Down-regulation of E-cadherin/catenin-mediated intercellular adhesion is known to be an important step in the acquisition of malignancy and metastasis. According to Baranwal [14], down-regulation of E-cadherin is associated with worse outcome and enhanced aggressiveness of the tumor. Klopp et al. [35] also stated that decreased expression of E-cadherin is associated with breast cancer progression and resistance to therapy. Finally, loss of E-cadherin expression is a hallmark of epithelial-mesenchymal transition (EMT), which is associated with a worse prognosis [16]. In contrast, up regulation of E-cadherin/catenin complex, which acts as a suppressor of tumor progression, has been accomplished with a series of agents, some of which can be used therapeutically [36].

Our finding of a significantly reduced expression of E-cadherin in multicentric/multifocal tumours underline and reinforce our view of a more aggressive behavior of this tumor type. Since loss of E-cadherin is a marker of EMT, it might be worthwhile to examine other EMT markers such as MMPs, which lead to E-cadherin degradation [37], or vimentin in multicentric/multifocal versus unifocal breast tumors.

MUC1 is a multifunctional epithelial glycoprotein known to be overexpressed in most epithelial cancers. MUC1 can promote proliferation and metastasis, whereas down regulation of MUC1 expression inhibits cell migration by inducing $\beta$-catenin relocation from the nucleus to the cytoplasm and increases E-cadherin/catenin complex formation [38]. In addition, MUC1 is coexpressed and complexed with STAT1 (Khodarev et al. [39]), and it is associated with decreased recurrence-free and overall survival. This may explain why intracellular expression of MUC1 is associated with worse prognosis [40], whereas 
membrane (or overall) expression of MUC1 is generally correlated with a better outcome [41].

Using the anti-MUC1 antibody mPankoMab, which recognizes a special, tumor-associated MUC1 epitope [19], we previously observed a correlation between MUC1 and the expression of the ER receptor [42]. In the present study, we did not observe differences in MUC1 expression between multicentric/multifocal and unifocal breast cancer $(\mathrm{p}=0.183)$. However, when looking at the histopathological grading, multicentric/multifocal carcinomas showed a statistically significant decrease in staining with increased histology grade $(\mathrm{p}=0.027)$ which was in contrast to the MUC1 expression in unifocal breast cancer of different grade.

According to the cytoplasmic PankoMab-staining no differences were found with respect to the histology grade. When looking at the overall survival (OS) the PankoMab epitope on the membrane was however associated with a better outcome, nevertheless only significant in G2 and G3 unifocal tumors ( $\mathrm{p}=0.038)$.

\section{Conclusions}

In summary, differences regarding tumo rbiology are obvious as fore the wnt signaling pathway might play an important role in unifocal tumors and the PankoMab epitope on the membrane associated with a better outcome in G2 and G3 unifocal tumors.

Due to the small collective used for this study, we have not confirmed and extended our earlier results which demonstrated that multicentric/multifocal tumors as compared to unifocal breast tumors correlate with a reduced survival and relapse-free interval (Additional file 1: Figure S1). Instead, we analyzed membrane associated breast cancer markers as molecules to discriminate with respect to focality between both entities. These results indicate that the breast tumor biology differs depending on focality and suggest a tendency for enhanced EMT in multicentric/ multifocal breast cancer. Further research is necessary on the tumor biology of multicentric and multifocal tumors.

\section{Additional file}

Additional file 1: Figure S1. Kaplan-Meier survival curves were drawn to compare Overall survival (OS) and relapse free survival (RFS) in unifocal and multicentric/multifocal tumors.

\section{Competing interest}

Uwe Karsten is an employee of Glycotope $\mathrm{GmbH}$ which mad and provided the PankoMab antibody. All other authors declare no competing interest.

\section{Authors' contributions}

TW designed the study and performed collection, analysis and interpretation of data and drafted the manuscript for publication. EH, CK and UK participated in the design of the study, and were involved in the immunhistochemistry. WJ, SH, ND, BR essentially were involved in the analysis and interpretation of the data and also approved the English. UJ, DD and FK performed participant inclusion, collected samples and contributed substantially to acquisition of data. DD helped substantially to draft the manuscript. All conceived of the study, participated in its design and coordination, helped with data interpretation and drafting of the manuscript. All authors read and approved the final manuscript.

\section{Acknowledgement}

We would like to thank Dr. Steven S. Witkin (Weill Cornell Medical College, New York, USA) for his help with the manuscript.

\section{Author details}

${ }^{1}$ Frauenklinik, Klinikum der Ludwig-Maximilians-Universität, Innenstadt, München, Germany. ${ }^{2}$ Frauenklinik, Heinrich-Heine-Universität, Düsseldorf, Germany. ${ }^{3}$ Pathologisches Institut, Ludwig-Maximilians-Universität, München, Germany. ${ }^{4}$ Frauenklinik, Klinikum der Ludwig-Maximilians-Universität, Großhadern, München, Germany. ${ }^{5}$ Glycotope GmbH, Berlin, Germany. 'Department of Gynecology and Obstetrics, Campus Innenstadt LudwigMaximilian-University Munich, Maistr. 11, Munich D-80337, Germany.

Received: 30 January 2013 Accepted: 22 July 2013

Published: 26 July 2013

\section{References}

1. Benson JR, Weaver DL, Mittra I, Hayashi M: The TNM staging system and breast cancer. Lancet Oncol 2003, 4(1):56-60.

2. Escobar PF, Patrick RJ, Rybicki LA, Weng DE, Crowe JP: The 2003 revised TNM staging system for breast cancer: results of stage re-classification on survival and future comparisons among stage groups. Ann Surg Oncol 2007, 14(1):143-147.

3. Veronesi U, Viale G, Rotmensz N, Goldhirsch A: Rethinking TNM: breast cancer TNM classification for treatment decision-making and research. Breast 2006, 15(1):3-8.

4. Bundred NJ: Prognostic and predictive factors in breast cancer. Cancer Treat Rev 2001, 27(3):137-142.

5. Dabakuyo TS, Bonnetain F, Roignot P, Poillot ML, Chaplain G, Altwegg T, Hedelin G, Arveux P: Population-based study of breast cancer survival in Cote d'Or (France): prognostic factors and relative survival. Ann Oncol 2008, 19(2):276-283.

6. Younes M, Lane M, Miller CC, Laucirica R: Stratified multivariate analysis of prognostic markers in breast cancer: a preliminary report. Anticancer Res 1997, 17(2B):1383-1390.

7. Weissenbacher TM, Zschage M, Janni W, Jeschke U, Dimpfl T, Mayr D, Rack $B$, Schindlbeck C, Friese K, Dian D: Multicentric and multifocal versus unifocal breast cancer: is the tumor-node-metastasis classification justified? Breast Cancer Res Treat 2010, 122(1):27-34.

8. Duraker N, Caynak ZC: Prognostic value of the 2002 TNM classification for breast carcinoma with regard to the number of metastatic axillary lymph nodes. Cancer 2005, 104(4):700-707.

9. Garcia Vilanova A, Sancho Merle MF, Vazquez Albaladejo C, Fuster Diana E, Cano Peral J: [Prognosis of stage II and III breast cancer in women and critique of various aspects of the TNM system]. Rev Esp Oncol 1980, 27(2):265-273.

10. Berx G, Nollet F, Van Roy F: Dysregulation of the E-cadherin/catenin complex by irreversible mutations in human carcinomas. Cell Adhes Commun 1998, 6(2-3):171-184.

11. Canavese G, Bernardi A, Candelaresi G, Lovadina P, Amerio S, Rossetti V, Rabagliati C, Berardengo E: Expression of the E-cadherin-catenins complex in sentinel node is related to tumor morphology but not to spread to nonsentinel nodes. Pathol Res Pract 2007, 203(7):517-523.

12. Kuroda H, Tamaru J, Takeuchi I, Ohnisi K, Sakamoto G, Adachi A, Kaneko K, Itoyama S: Expression of E-cadherin, alpha-catenin, and beta-catenin in tubulolobular carcinoma of the breast. Virchows Arch 2006, 448(4):500-505.

13. Niu LG, He JJ, Wang K, Zhang W, Zhou C: Abnormal expression of beta-catenin and E-cadherin in Her2-positive breast cancer and its implications. Nan Fang Yi Ke Da Xue Xue Bao 2009, 29(11):2237-2240.

14. Baranwal S, Alahari SK: Molecular mechanisms controlling E-cadherin expression in breast cancer. Biochem Biophys Res Commun 2009, 384(1):6-11.

15. Schmalhofer O, Brabletz S, Brabletz T: E-cadherin, beta-catenin, and ZEB1 in malignant progression of cancer. Cancer Metastasis Rev 2009, 28(1-2):151-166. 
16. Zheng G, Lyons JG, Tan TK, Wang Y, Hsu TT, Min D, Succar L, Rangan GK, Hu $M$, Henderson BR, et al: Disruption of E-cadherin by matrix metalloproteinase directly mediates epithelial-mesenchymal transition downstream of transforming growth factor-beta1 in renal tubular epithelial cells. Am J Pathol 2009, 175(2):580-591.

17. Price MR, Rye PD, Petrakou E, Murray A, Brady K, Imai S, Haga S, Kiyozuka Y Schol D, Meulenbroek MF, et al: Summary report on the ISOBM TD-4 Workshop: analysis of 56 monoclonal antibodies against the MUC1 mucin. San Diego, Calif., November 17-23, 1996. Tumour Biol 1998, 19(Suppl 1):1-20.

18. Kufe DW: Mucins in cancer: function, prognosis and therapy. Nat Rev Cancer 2009, 9(12):874-885.

19. Danielczyk A, Stahn R, Faulstich D, Loffler A, Marten A, Karsten U, Goletz S: PankoMab: a potent new generation anti-tumour MUC1 antibody. Cancer Immunol Immunother 2006, 55(11):1337-1347.

20. Dian D, Janni W, Kuhn C, Mayr D, Karsten U, Mylonas I, Friese K, Jeschke U: Evaluation of a novel anti-mucin 1 (MUC1) antibody (PankoMab) as a potential diagnostic tool in human ductal breast cancer; comparison with two established antibodies. Onkologie 2009, 32(5):238-244.

21. Prasad CP, Rath G, Mathur S, Bhatnagar D, Parshad R, Ralhan R: Expression analysis of E-cadherin, slug and GSK3beta in invasive ductal carcinoma of breast. BMC Cancer 2009, 9:325.

22. Takahashi-Yanaga F, Kahn M: Targeting Wnt signaling: can we safely eradicate cancer stem cells? Clin Cancer Res 2010, 16(12):3153-3162.

23. Sobin $\mathrm{LH}$, Hermanek $\mathrm{P}$, Hutter RV: TNM classification of malignant tumors. a comparison between the new (1987) and the old editions. Cancer 1988, 61(11):2310-2314.

24. Karsten U, Von Mensdorff-Pouilly S, Goletz S: What makes MUC1 a tumor antigen? Tumour Biol 2005, 26(4):217-220.

25. Elston CW, Ellis IO: Pathological prognostic factors in breast cancer. I. the value of histological grade in breast cancer: experience from a large study with long-term follow-up. Histopathology 1991, 19(5):403-410.

26. Vlastos G, Rubio IT, Mirza NQ, Newman LA, Aurora R, Alderfer J, Buzdar AU, Singletary SE: Impact of multicentricity on clinical outcome in patients with T1-2, N0-1, M0 breast cancer. Ann Surg Oncol 2000, 7(8):581-587.

27. Tot T: Early and more advanced unifocal and multifocal breast carcinomas and their molecular phenotypes. Clin Breast Cancer 2011, 11(4):258-263.

28. Tot T, Pekar G: Multifocality in "basal-like" breast carcinomas and its influence on lymph node status. Ann Surg Oncol 2011, 18(6):1671-1677.

29. Tot T, Pekar G, Hofmeyer S, Gere M, Tarjan M, Hellberg D, Lindquist D: Molecular phenotypes of unifocal, multifocal, and diffuse invasive breast carcinomas. Patholog Res Int 2010, 2011:480960.

30. Boyages J, Jayasinghe UW, Coombs N: Multifocal breast cancer and survival: each focus does matter particularly for larger tumours. Eur J Cancer 2010, 46(11):1990-1996.

31. Pekar G, Hofmeyer S, Tabar L, Tarjan M, Chen TH, Yen AM, Chiu SY, Hellberg $D$, Gere M, Tot T: Multifocal breast cancer documented in large-format histology sections: long-term follow-up results by molecular phenotypes. Cancer 2013, 119(6):1132-1139.

32. Bassarova AV, Torlakovic E, Sedloev T, Hristova SL, Trifonov DV, Nesland JM: Simultaneous bilateral breast carcinoma: histopathological characteristics and CD44/catenin-cadherin profile. Histol Histopathol 2005, 20(3):791-799.

33. Mohinta $S$, Wu H, Chaurasia P, Watabe K: Wnt pathway and breast cancer. Front Biosci 2007, 12:4020-4033.

34. Lopez-Knowles E, Zardawi SJ, McNeil CM, Millar EK, Crea P, Musgrove EA, Sutherland RL, O'Toole SA: Cytoplasmic localization of beta-catenin is a marker of poor outcome in breast cancer patients. Cancer Epidemiol Biomarkers Prev 2010, 19(1):301-309.

35. Klopp AH, Lacerda L, Gupta A, Debeb BG, Solley T, Li L, Spaeth E, Xu W, Zhang X, Lewis MT, et al: Mesenchymal stem cells promote mammosphere formation and decrease E-cadherin in normal and malignant breast cells. PLoS One 2010, 5(8):e12180.

36. Debruyne $\mathrm{P}$, Vermeulen S, Mareel M: The role of the E-cadherin/catenin complex in gastrointestinal cancer. Acta Gastroenterol Belg 1999, 62(4):393-402.

37. Bukholm IR, Nesland JM, Bukholm G: Expression of adhesion proteins E-cadherin, alpha-catenin, beta-catenin and gamma-catenin is different in T1 and T2 breast tumours. Pathology 2006, 38(5):403-407.
38. Yuan Z, Wong S, Borrelli A, Chung MA: Down-regulation of MUC1 in cancer cells inhibits cell migration by promoting E-cadherin/catenin complex formation. Biochem Biophys Res Commun 2007, 362(3):740-746.

39. Khodarev N, Ahmad R, Rajabi H, Pitroda S, Kufe T, McClary C, Joshi MD, MacDermed D, Weichselbaum R, Kufe D: Cooperativity of the MUC1 oncoprotein and STAT1 pathway in poor prognosis human breast cancer. Oncogene 2010, 29(6):920-929.

40. De Oliveira JT, Pinho SS, De Matos AJ, Hespanhol V, Reis CA, Gartner F: MUC1 expression in canine malignant mammary tumours and relationship to clinicopathological features. Vet J 2009, 182(3):491-493.

41. van der Vegt B, De Roos MA, Peterse JL, Patriarca C, Hilkens J, De Bock GH, Wesseling J: The expression pattern of MUC1 (EMA) is related to tumour characteristics and clinical outcome of invasive ductal breast carcinoma. Histopathology 2007, 51(3):322-335.

42. De Roos MA, van der Vegt B, Peterse JL, Patriarca C, De Vries J, De Bock GH, Wesseling J: The expression pattern of MUC1 (EMA) is related to tumour characteristics and clinical outcome in 'pure' ductal carcinoma in situ of the breast. Histopathology 2007, 51(2):227-238.

doi:10.1186/1471-2407-13-361

Cite this article as: Weissenbacher et al.: Multicentric and multifocal versus unifocal breast cancer: differences in the expression of E-cadherin suggest differences in tumor biology. BMC Cancer 2013 13:361.

\section{Submit your next manuscript to BioMed Central and take full advantage of:}

- Convenient online submission

- Thorough peer review

- No space constraints or color figure charges

- Immediate publication on acceptance

- Inclusion in PubMed, CAS, Scopus and Google Scholar

- Research which is freely available for redistribution 